\title{
EDITORIAL
}

\section{Toward a developmental psychopathology approach to borderline personality disorder}

\author{
MARK F. LENZENWEGER ${ }^{a}$ AND DANTE CICCHETTI ${ }^{b}$ \\ ${ }^{a}$ State University of New York at Binghamton; and ${ }^{b}$ Mt. Hope Family Center, \\ University of Rochester, and Institute of Child Development, University of Minnesota
}

Borderline personality disorder (BPD) is one of the more vexing forms of psychopathology for researchers and clinicians alike to understand. Currently, the etiology and pathogenesis of the disorder remain unclear; however, steady progress has been made in several important areas that bear upon informing a more complete understanding of this condition. Long known to clinicians in office practice and mental health staff at clinics and hospitals, BPD is a heterogenous phenotype characterized by a complex array of features such as emotional dysregulation, interpersonal dysfunction, impulsivity, self-damaging behaviors, excessive anger and fear, and identity disturbance (American Psychiatric Association, 1994). The individual afflicted with BPD frequently experiences substantial social and occupational impairment, as well as frequent emotional turmoil. Those individuals who spend considerable time with a BPD-affected person, such as parents, partners, spouses, children, coworkers, and others, also encounter appreciable stresses and strains associated with the impact of BPD on the family, social, and occupational milieu.

At this time, epidemiological estimates reveal point prevalences for the disorder at between 1.3 (Lenzenweger, Loranger, Korfine,

Address correspondence and reprint requests to: Mark F. Lenzenweger, SUNY Binghamton, Science IV, P.O. Box 6000, Binghamton, NY 13902-6000; E-mail: mlenzen@ binghamton.edu.
\& Neff, 1997) and 0.7\% (Torgersen, Kringlen, \& Cramer, 2001). Thus, it appears that approximately 1 in every $100-200$ people is affected with BPD. (By comparison, schizophrenia and bipolar disorder each affect about 1 in every 100 people.)

\section{Phases of Inquiry into BPD}

The history of research and clinical exploration of BPD can be divided roughly into four phases, beginning with clinical observation through model-guided experimental studies. The earliest phase (prior to the 1960s) focused on clinical descriptions of the disorder and the efforts of practitioners to fit this entity into existing diagnostic conceptualizations. Thus, early clinicians (e.g., Knight, 1953; Stern, 1938) struggled to determine if what would become known as BPD had any connection to psychosis, particularly schizophrenia.

The second phase (1960s and 1970s) consisted largely of "description and emergence of explicit diagnostic criteria" for BPD. This phase began with the rich clinical insights and theoretical speculations of Kernberg (1967), as well as the creative early efforts to rigorously explore the latent structure of behavioral and psychological indicators of BPD-related behaviors (Grinker, Werble, \& Drye, 1968). This phase culminated with the publication of two highly influential papers. The first is the well-known literature review by Gunderson and Singer (1975), which pro- 
vided an organizational focus for the prior descriptive efforts, integrating phenomenological issues with psychological testing findings. The second paper by Spitzer, Endicott, and Gibbon (1979) detailed the background and development of the diagnostic criteria for BPD that would be reflected subsequently in the DSM-III (American Psychiatric Association, 1980). Each of these papers has been cited over 400 times.

The third phase of work can be described as being largely focused on the empirical evaluation of the proposed diagnostic criteria for BPD in the DSM-III and the development of assessment technologies. From the standpoint of assessment, new instrumentation was developed to assess the diagnostic criteria for BPD as specified in the DSM-III, and there was a considerable focus on matters related to reliability. This occurred because, although the $D S M$-III listed explicit criteria for the disorder, the system was not operationalized. Thus, it was left up to instrument developers to discern the meaning of the DSM features and to create a reliable method for their assessment. It is not surprising that at this time (1980s) much work was devoted to the development of high-quality diagnostic instruments for the assessment of all Axis II disorders. Thus, this period saw the emergence of the International Personality Disorders Examination (IPDE; Loranger, 1988, 1999), the Structured Clinical Interview for DSM-III-R Axis II Disorders (SCID-II; Spitzer, Williams, \& Gibbon, 1987), and the Structured Interview for DSM-III Personality (Pfohl, Stangl, \& Zimmerman, 1983). This period also witnessed significant interest in the patterns of covariation among disorders (comorbidity), as well as evaluation of the predictive value of specific symptoms in the personality disorders (PDs). BPD was very much a focus in all of these efforts; assessment of BPD was moved forward during this period, and there were the beginnings of genuine validation research. The latter point was exemplified in a report by Loranger, Oldham, and Tulis (1982), where it was noted that BPD was familial and did not reside on the "border" of psychosis. The impact of the DSM-III on the rate at which BPD was diagnosed was unmistakable in that the rate of the diagnosis increased dramatically after 1980 (Loranger, 1990).

The third phase of research (1990s) involved work directed at specifying the correlates of BPD. For example, during this time an interest in cognition and memory in BPD began to emerge, with the appearance of a number of studies that characterized the neuropsychological functioning of BPD (for a review see Fertuck, Lenzenweger, Clarkin, Hoermann, \& Stanley, in press). The focus on trauma histories among patients with BPD was brought more clearly into focus. The normal personality correlates of the BPD construct were specified such that it was confirmed that observing clinicians were quite accurate in their descriptions of patients with BPD as low on agreeableness and high on neuroticism. This phase of research would eventually give way to model-guided research that sought to examine the processes and mechanisms involved in BPD, the fourth and current phase.

The fourth phase of research is characterized, in our minds, by efforts to better elucidate the basic core processes of BPD. We believe this phase of research, beginning in 2000, has already shown a preference for a focus on process and mechanism in BPD. The research that is beginning to appear seeks to specify the nature of a dysfunctional process in BPD, articulate those parameters that define the process and its variability, and, ideally, place these findings within a context that will enhance our understanding of the development and emergence of BPD. A seminal investigation that typifies this mode of inquiry was conducted by Korfine and Hooley (2000), and it focused on directed forgetting in BPD. In this study it was found that BPD subjects, whether drawn from clinical settings or from the community, remembered "borderline" words (e.g., abandon, alone, enraged, hate, misunderstood, reject, suicidal, uncaring) that they were instructed to forget. These findings provided an empirical basis for theoretical conjectures regarding the cognitive mechanisms through which a disinhibitory process exerts its influence. We argue that investigations of this form will typify the current phase of research on BPD and, by their nature, advance our understanding of the com- 
ponent processes that underlie this disorder, and the research corpus will soon transcend mere description of the correlates of BPD.

\section{Future Directions}

Although it is clearly evident that the work in this area has moved beyond largely clinical observations and treatment-related speculation to a mode that will engender greater clarity with respect to etiology, pathogenesis, and development, there remains much to explore in the territory of BPD. We therefore want to address several issues that require greater empirical investigation, as well as areas in need of enhanced theoretical integration. To begin, we still do not have an adequate portrait of the life-course trajectory of those afflicted with BPD. Although clinical lore suggests that patients with BPD remain largely stable throughout most of their adult lives, perhaps with some showing a diminution of symptomatology in midlife, the existing empirical database in support of this suggestion remains thin. The need for ongoing prospective multiwave longitudinal studies that are properly designed and contain necessary methodological safeguards should be obvious (e.g., Lenzenweger, Johnson, \& Willett, 2004).

A second focus area requires resolving the underlying structure and natural organization of BPD. It is quite clear that the DSM approach to the diagnosis of BPD yields a phenotype that is characterized by considerable heterogeneity. This means that there can be great variability across the population of individuals diagnosed with BPD. This heterogeneity may represent our flawed efforts to define a complex phenotype, it may reflect a diversity of underlying pathological processes, or both. The field is in genuine need of wellconducted latent structure analyses of the BPD phenotype, preferably using techniques that can efficiently sort individuals into meaningful categories (e.g., finite mixture modeling, latent class analysis). A third focus in the study of BPD needs to be one that draws upon the rich and rigorous methods of the experimental psychology laboratory (Bornstein, 2003; Lenzenweger \& Hooley, 2003). In utilizing the laboratory approach, one not only gains the leverage provided by precise methods of data collection, but one also can normally collect data in a "counting" manner rather than a "rating" manner. The benefits of counting data, with associated desirable ratio-scale characteristics, are well known (Maher, 2003). Thus, for example, we encourage efforts to assess impulsive behaviors experimentally in subjects rather than have investigators rely upon rating impulsivity or having subjects report on themselves with questionnaires. Ideally, from a developmental psychopathology perspective, such assessments should be done over time and meaningful developmental epochs. Within this context, we also argue for a careful application of the powerful new laboratory methods of neuroimaging (positron emission tomography, functional magnetic resonance imaging, and other approaches) to substantive questions regarding BPD. Clearly, neuroimaging techniques need to be brought to bear upon many aspects of emotional and cognitive processing in BPD; however, we urge that this research should emphasize mechanism and process, rather than mere articulation of the "neural activation correlates" of BPD. Here, we wholly embrace the spirit of Kosslyn's question to the field of psychological science: "When neuroimaging is the answer, what is the question?" (Kosslyn, 1999).

A fourth area in which we believe research should be encouraged is in the area of endophenotypes (Gottesman \& Gould, 2003; Gottesman \& Hanson, 2005). The endophenotype, according to Gottesman and Gould (2003), is a measurable component unseen by the unaided naked eye along the pathway between distal genotype and disease. It may be neurophysiological, endocrinological, neuroanatomical, cognitive, or neuropsychological (including configured self-report data) in nature. Moreover, the endophenotype likely represents a simpler clue to genetic underpinnings than the disease syndrome itself. The endophenotype concept is not new to psychopathology research, as it has been used extensively for years in the schizophrenia and schizotypy realm (cf., Gottesman \& Shields, 1972; Lenzenweger \& Loranger, 1989); however, it is a relative newcomer to the BPD research world. 
We argue that for research on BPD to advance, endophenotypes will need to be incorporated into future studies. They will provide needed leverage to genomic, neuroimaging, neurobiological, and psychological investigations alike.

A fifth area in need of continued development and integration concerns our models of PD (Lenzenweger \& Clarkin, 2005), particularly our understanding of neurobehavioral systems. A neurobiological understanding of personality and PDs represents a fruitful avenue not only for model development, but also for the generation of hypotheses for other studies. It is now possible to see powerful links between underlying neurobehavioral systems, indicators of those systems within the personality realm, and PD (see Depue \& Lenzenweger, 2005). We suggest that these links should be tested where possible and that neurobehavioral models should form the basis of laboratory-based inquiries.

There also are several research areas that show considerable promise for elucidating our understanding of BPD. We believe that a developmental perspective is necessary to achieve the genuine progress that can be made in these domains.

- Specifically, there is a clear need to articulate and understand those factors that might contribute to the maintenance of BPD over the life course, quite apart from those that might contribute to its etiology.

- A fuller understanding of the role played by trauma in the development of BPD is needed. For example, if trauma is causative of BPD in nature, then how might one go about studying the unfolding of this process, mindful of the complexities such work would pose?

- As psychopathology research continues to incorporate genomic methods into its research strategy, it would be useful to link such genomic methods with laboratory investigations in search of endophenotypes (Gottesman \& Gould, 2003).

Finally, there are a number of research areas that, if approached from a developmental psychopathology perspective, would make major contributions toward advancing our understanding of BPD. These include the following:
- In keeping with a developmental psychopathology framework (Cicchetti \& Cohen, in press-a, in press-b, in press-c), the existing knowledge of the potential putative precursors to BPD should be included in the development of the measurement batteries utilized in future investigations of the etiology, course, and consequences of BPD. These include assessing the temperamental characteristics of low effortful control and negative affect, relational aggression, emotion regulation and dysregulation, impulsivity, affect instability, and relationship dysfunction.

- To develop a thorough and comprehensive understanding of adaptive and maladaptive functioning in individuals with BPD, it is essential that scientists increasingly incorporate a multiple levels of analysis perspective into their research (Cicchetti \& Blender, 2004; Cicchetti \& Dawson, 2002).

- The systems theory concepts of equifinality and multifinality should become incorporated into the research designs of studies investigating the differential pathways to BPD, as well as the diversity of outcomes experienced (Cicchetti \& Rogosch, 1996).

- There is a strong need to be able to study BPD before it emerges. What populations should be targeted to enhance the likelihood of observing BPD at greater than population prevalence rates (e.g., maltreated children and adolescents; offspring of parents with BPD)?

- What are the earlier precursors to BPD? How can prodromal signs be identified?

- It is important to understand the reasons why there is a greater female prevalence of BPD. Is this related to genetic differences, different socialization pressures, greater rates of sexual abuse, or the utilization of differential diagnostic practices among practitioners for men and women?

- How do possible genetic predispositions to BPD become realized?

- Are there components of BPD that constitute more biological potentialities (e.g., impulsivity, excess anger and fear, emotion regulation problems), as well as other components that have more experiential influence (e.g., identity disturbance, interpersonal dysfunction)? 
- There is a need to understand the experiential contributors to BPD phenomenology. In this regard, it is important to consider the developmental experience of persons with BPD to understand how the PD evolved. For example, how does experience contribute to or intensify problems in emotion regulation, interpersonal dysfunction, excess anger and fear, and identity disturbance?

- It is essential to examine how development influences the neurobiological features that purportedly are atypical in persons with $\mathrm{BPD}$. A related question is how does experience influence neurobiological processes and gene expression in individuals with BPD? In contemporary perspective, experience is broadly construed to include not only external social and psychological events, but also, for example, internal events such as the effects of psychopathology, trauma, abuse, and the action of hormones (Boyce et al., 1998; Cicchetti \& Walker, 2003). Just as gene expression alters social behavior, so, too, do social experiences exert actions on the brain by feeding back upon it to modify gene expression and brain structure, function, and organization. Alterations in gene expression induced by social experiences produce changes in neuronal and synaptic connections and, thus, in the function of nerve cells (Kandel, 1998, 1999). Such neuronal and synaptic modifications not only exert a prominent role in initiating and maintaining the behavioral changes that are provoked by experience but also contribute to the biological bases of individuality, including personality organization. Given the bidirectional interplay between biology and experience, researchers must move away from "main effect" thinking that the neurobiological process is a singular cause of BPD.
- What role do the normal neurobiological developmental changes that take place during late adolescence and early adulthood (e.g., pubertal increases in gonadal hormones during adolescence; developmental transformations in prefrontal cortex and limbic brain regions; continued myelination of intracortical connections) play in potentiating the development of BPD in psychologically and genetically vulnerable individuals (Cicchetti \& Rogosch, 2002; Spear, 2000)?

- How do object relations/attachment organization, based on history of experience, contribute to BPD? What biological vulnerabilities might be necessary to eventuate in $\mathrm{BPD}$, given an early history of trauma or dysfunctional attachments?

- Is BPD primarily a disorder of early adulthood onset? What makes the developmental challenges of this period (e.g., identity, intimacy, relationships) so difficult?

Individuals with BPD contribute significantly to the burden of mental illness and drain resources from the medical and mental health communities. Moreover, given the substantial social stigma directed toward individuals who have been diagnosed with this disorder, efforts directed toward elucidating the etiology and course of BPD are critical. Ultimately, such efforts can inform treatment and minimize the individual, familial, and societal burden of this illness.

We thank the contributors to this Special Issue for their efforts in informing our understanding of BPD, and we hope that their work will inspire others in the field to embark on research with this fascinating and challenging population.

\section{References}

American Psychiatric Association. (1980). Diagnostic and statistical manual of mental disorders (3rd ed.). Washington, DC: Author.

American Psychiatric Association. (1994). Diagnostic and statistical manual of mental disorders (4th ed.). Washington, DC: Author.

Bornstein, R. F. (2003). Behaviorally referenced experimentation and symptom validation: A paradigm for

21 st century personality disorder research. Journal of Personality Disorders, 17, 1-18.

Boyce, W. T., Frank, E., Jensen, P. S., Kessler, R. C., Nelson, C. A., Steinberg, L., \& The MacArthur Foundation Research Network on Psychopathology and Development. (1998). Social context in developmental psychopathology: Recommendations for future research from The MacArthur Network on Psychopa- 
thology and Development. Development and Psychopathology, 10, 143-164.

Cicchetti, D., \& Blender, J. A. (2004). A multiple-levelsof-analysis approach to the study of developmental processes in maltreated children. Proceedings of the National Academy of Sciences, 101, 17325-17326.

Cicchetti, D., \& Cohen, D. (Eds.). (in press-a). Developmental psychopathology (2nd ed.): Theory and method (Vol. 1). New York: Wiley.

Cicchetti, D., \& Cohen, D. (Eds.). (in press-b). Developmental psychopathology (2nd ed.): Developmental neuroscience (Vol. 2). New York: Wiley.

Cicchetti, D., \& Cohen, D. (Eds.). (in press-c). Developmental psychopathology (2nd ed.): Risk, disorder, and adaptation (Vol. 3). New York: Wiley.

Cicchetti, D., \& Dawson, G. (Eds.). (2002). Multiple levels of analysis. Development and Psychopathology, 14, 417-666.

Cicchetti, D., \& Rogosch, F. A. (1996). Equifinality and multifinality in developmental psychopathology. Development and Psychopathology, 8, 597-600.

Cicchetti, D., \& Rogosch, F. A. (2002). A developmental psychopathology perspective on adolescence. Journal of Consulting and Clinical Psychology, 70, 6-20.

Cicchetti, D., \& Walker, E. F. (Eds.). (2003). Neurodevelopmental mechanisms in psychopathology. New York: Cambridge University Press.

Depue, R. A., \& Lenzenweger, M. F. (2005). A neurobehavioral model of personality disturbance. In J. F. Clarkin \& M. F. Lenzenweger (Eds.), Major theories of personality disorder (2nd ed., pp. 391-453). New York: Guilford Press.

Fertuck, E. A., Lenzenweger, M. F., Clarkin, J. F., Hoermann, H., \& Stanley, B. (in press). Executive neurocognition, memory systems, and borderline personality disorder. Clinical Psychology Review.

Gottesman, I. I., \& Gould, T. D. (2003). The endophenotype concept in psychiatry: Etymology and strategic intentions. American Journal of Psychiatry, 160, 636-645.

Gottesman, I. I., \& Hanson, D. R. (2005). Human development: Biological and genetic processes. Annual Review of Psychology, 56, 263-286.

Gottesman, I. I., \& Shields, J. (1972). Schizophrenia and genetics: A twin study vantage point. New York: Academic Press.

Grinker, R. R., Werble, R., \& Drye, R. C. (1968). The borderline syndrome: A behavioral study of ego functions. New York: Basic Books.

Gunderson, J. G., \& Singer, M. T. (1975). Defining borderline patients: An overview. American Journal of Psychiatry, 132, 1-10.

Kandel, E. R. (1998). A new intellectual framework for psychiatry. American Journal of Psychiatry, 155, 475-469.

Kandel, E. R. (1999). Biology and the future of psychoanalysis: A new intellectual framework for psychiatry revisited. American Journal of Psychiatry, 156, 505-524.

Kernberg, O. F. (1967). Borderline personality organization. Journal of the American Psychoanalytic Association, 15, 641-685.

Knight, R. R. (1953). Borderline states in psychoanalytic psychiatry and psychology. Bulletin of the Menniger Clinic, 17, 1-12

Korfine, L., \& Hooley, J. M. (2000). Directed forgetting of emotional stimuli in borderline personality disorder. Journal of Abnormal Psychology, 109, 214-221.

Kosslyn, S. M. (1999). If neuroimaging is the answer, what is the question? Transactions of the Royal Society of London Series B-Biological Sciences, 354, 1283-1294.

Lenzenweger, M. F., \& Clarkin, J. F. (Eds.). (2005). Major theories of personality disorder (2nd ed.). New York: Guilford Press.

Lenzenweger, M. F., \& Hooley, J. M. (Eds.). (2003). Principles of experimental psychopathology: Essays in honor of Brendan A. Maher. Washington, DC: American Psychological Association.

Lenzenweger, M. F., Johnson, M. D., \& Willett, J. B. (2004). Individual growth curve analysis illuminates stability and change in personality disorder features: The Longitudinal Study of Personality Disorders. Archives of General Psychiatry, 61, 1015-1024.

Lenzenweger, M. F., \& Loranger, A. W. (1989). Detection of familial schizophrenia using a psychometric measure of schizotypy. Archives of General Psychiatry, 46, 902-907.

Lenzenweger, M. F., Loranger, A. W., Korfine, L., \& Neff, C. (1997). Detecting personality disorders in a nonclinical population: Application of a 2-stage for case identification. Archives of General Psychiatry, $54,345-351$.

Loranger, A. (1988). The personality disorder examination $(P D E)$ manual. Yonkers, NY: DV Communications.

Loranger, A. (1990). The impact of DSM-III on diagnostic practice in a university hospital: A comparison of DSM-II and DSM-III in 10,914 patients. Archives of General Psychiatry, 47, 672-675.

Loranger, A. W. (1999). International personality disorder examination: DSM-IV and ICD-10 interviews. Odessa, FL: Psychological Assessment Resources, Inc.

Loranger, A. W., Oldham, J. M., \& Tulis, E. H. (1982). Familial transmission of DSM-III borderline personality disorder. Archives of General Psychiatry, 39, 795-799.

Maher, B. A. (2003). Psychopathology and delusions: Reflections on methods and models. In M. F. Lenzenweger \& J. M. Hooley (Eds.), Principles of experimental psychopathology: Essays in honor of Brendan A. Maher (pp. 9-28). Washington, DC: American Psychological Association.

Pfohl, B., Stangl, D., \& Zimmerman, M. (1983). Structured Interview for DSM-III personality. Iowa City, IA: University of Iowa.

Spear, L. P. (2000). The adolescent brain and age-related behavioral manifestations. Neuroscience and Behavioral Reviews, 24, 417-463.

Spitzer, R. L., Endicott, J., \& Gibbon, M. (1979). Crossing the border into borderline personality and borderline schizophrenia: The development of criteria. Archives of General Psychiatry, 36, 17-24.

Spitzer, R., Williams, J., \& Gibbon, M. (1987). Structured Clinical Interview for DSM-III-R personality disorders (SCID-II). New York: New York State Psychiatric Institute.

Stern, A. (1938). Psychoanalytic investigation of and therapy in the border land group of neuroses. Psychoanalytic Quarterly, 7, 467-489.

Torgersen, S., Kringlen, E., \& Cramer, V. (2001). The prevalence of personality disorders in a community sample. Archives of General Psychiatry, 58, 590-596. 